\title{
A COMBINATORIAL PROBLEM AND CONGRUENCES FOR THE RAYLEIGH FUNCTION
}

\author{
F. T. HOWARD
}

Abstract. Let $z$ be a positive integer and let $m$ be the number of nonzero terms in the base 2 expansion of $z$. Define $f(z, s)$ as the number of positive integers $r \leqq z / 2$ such that the number of nonzero terms in the base 2 expansion of $r$ plus the number of nonzero terms in the base 2 expansion of $z-r$ is equal to $m+s$. We find formulas for $f(z, s)$ and show how these formulas can be used in proving congruences for the Rayleigh function.

1. Introduction. We shall assume that

$$
z=2^{e_{1}}+\cdots+2^{e_{m}}, \quad e_{1}>\cdots>e_{m} \geqq 0 .
$$

For $s \geqq 0$ define $f(z, s)$ as the number of positive integers $r \leqq z / 2$ such that if

$$
\begin{aligned}
r=2^{b_{1}}+\cdots+2^{b_{v}}, & b_{1}>\cdots>b_{0} \geqq 0, \\
z-r=2^{c_{1}}+\cdots+2^{c_{w}}, & c_{1}>\cdots>c_{w} \geqq 0, \\
& \text { then } v+w=m+s .
\end{aligned}
$$

In $\S 4$ we point out that $f(z, s)$ is equal to the number of binomial coefficients $\left(\begin{array}{r}2 \\ )\end{array}\right), 0<r \leqq z / 2$, divisible by $2^{s}$ but not by $2^{s+1}$. The main purpose of this paper is to consider the problem of evaluating $f(z, s)$ for $s \geqq 0$ and to show how this function can be used for proving congruences for the Rayleigh function, $\sigma_{2 n}(\nu)$. We note that in [2] it was proved that $f(z, 0)=2^{m-1}-1$.

The Rayleigh function has been the subject of a number of papers by Kishore (see [3] and [4] for example). It can be defined by means of the recurrence formula

$$
(\nu+n) \sigma_{2 n}(\nu)=\sum_{i=1}^{n-1} \sigma_{2 i}(\nu) \sigma_{2 n-2 i}(\nu),
$$

where $\sigma_{2}(\nu)=1 / 4(\nu+1)$. It is known that

$$
\begin{aligned}
\sigma_{2 n}(1 / 2) & =(-1)^{n-1} 2^{2 n-1} B_{2 n} /(2 n) !, \\
\sigma_{2 n}(-1 / 2) & =(-1)^{n} 2^{2 n-2} G_{2 n} /(2 n) !
\end{aligned}
$$

Received by the editors December 10, 1969.

AMS 1968 subject classifications. Primary 0510, 1006, 1007.

Key words and phrases. Rayleigh function, Bernoulli numbers, compositions of integers, enumerating generating function. 
where $B_{2 n}$ is the $2 n$th Bernoulli number and $G_{n}=2\left(1-2^{n}\right) B_{n}$.

In this paper we prove congruences $(\bmod 8)$ and $(\bmod 16)$ for $2^{y} \sigma_{2 n}(a / b)$, when neither the numerator nor denominator of $2^{y} \sigma_{2 n}(a / b)$ is divisible by 2 .

2. A combinatorial problem. The notation of the introduction will be used in this section, and we will make use of the function $\alpha(j)$ defined by means of

$$
\begin{aligned}
\alpha(j) & =2 \text { if } s+m=2 j \\
& =1 \text { if } s+m>2 j .
\end{aligned}
$$

THEOREM 2.1. If $e_{i}-e_{i+1}>s>0$ for $i=1, \cdots, m-1$ and $e_{m} \geqq s$, then

$$
f(z, s)=\sum_{j=1}^{m}\left(\begin{array}{l}
m \\
j
\end{array}\right)\left(\begin{array}{l}
s-1 \\
j-1
\end{array}\right) \alpha(j) 2^{s+m-2 j-1}
$$

Proof. To find $r$ and $z-r$ satisfying (1.1) we first pick $j$ elements from $e_{1}, \cdots, e_{m}$. Call them $d_{1}, \cdots, d_{j}$. Consider any composition of $s$ into $j$ parts (see $[5$, p. 107]):

$$
s=s_{1}+\cdots+s_{j}
$$

Then for $i=1, \cdots, j$ we have

$$
2^{d_{i}}=2^{d_{i}-s_{i}}+\sum_{h=0}^{s_{i}-1} 2^{d_{i}-s_{i}+h} .
$$

The exponents $b$ and $c$ of (1.1) will be chosen from the $s+j$ numbers $d_{i}-s_{i}+h$ and the $m-j e$ 's which were not picked. There are $2^{m+s-2 j}$ ways to divide these numbers between $r$ and $z-r$. There are $\left(\begin{array}{l}m \\ j\end{array}\right)$ ways to pick $d_{1}, \cdots d_{j}$ and there are $\left(\begin{array}{c}8-1 \\ j-1\end{array}\right)$ compositions of $s$ into $j$ parts $[5$, p. 124]. We divide our final answer by 2 (except when $s+m=2 j$ ) to eliminate $r>z / 2$.

THEOREM 2.2. If $e_{i}-e_{i+1}>s>0$ for $q$ terms $e_{i}$, if $e_{i}-e_{i+1}=s$ for $m-q-1$ terms and $e_{m} \geqq s$, then

$$
f(z, s)=(q+1) 2^{s+m-3}+\sum_{j=2}^{m}\left(\begin{array}{c}
m \\
j
\end{array}\right)\left(\begin{array}{c}
s-1 \\
j-1
\end{array}\right) \alpha(j) 2^{s+m-2 j-1} .
$$

THEOREM 2.3. If $e_{i}-e_{i+1}>s>0$ for $q$ terms $e_{i}$, if $e_{i}-e_{i+1}=s$ for $m-q-1$ terms and $e_{m}=u, 0 \leqq u<s$, then 


$$
\begin{aligned}
f(z, s)=q 2^{s+m-3} & +\sum_{j=2}^{m}\left(\begin{array}{c}
m \\
j
\end{array}\right)\left(\begin{array}{c}
s-1 \\
j-1
\end{array}\right) \alpha(j) 2^{s+m-2 j-1} \\
& -\sum_{j=2}^{s-u}\left(\begin{array}{c}
m-1 \\
j-1
\end{array}\right)\left(\begin{array}{c}
s-u-1 \\
j-1
\end{array}\right) \alpha(j) 2^{s+m-2 j-1} .
\end{aligned}
$$

Proof. We use the method of Theorem 2.1. Pick $j$ terms from $e_{1}, \cdots, e_{m}$ and call them $d_{1}, \cdots, d_{j}$. The term $q 2^{s+m-3}$ in the formula corresponds to the case $j=1$. For $2 \leqq j \leqq m$ we proceed exactly as we did in Theorem 2.1. We observe, however, that certain compositions of $s$ into $j$ parts will not be allowed when $2 \leqq j \leqq s-u$ and $e_{m}$ is picked as one of the $d$ 's. When this happens, there are $\left(\begin{array}{c}m-1 \\ j-1\end{array}\right)$ ways of picking the remaining $d$ 's, and we need to find the number of compositions of $s$ in to $j$ parts $s_{1}+\cdots+s_{j}$ such that $s_{1}>u$. An enumerating generating function for such compositions is

$$
\left(x^{u+1}+x^{u+2}+\cdots\right)\left(x+x^{2}+x^{3}+\cdots\right)^{j-1} .
$$

From this we determine the number of such compositions to be $\left(\begin{array}{c}s-u-1 \\ j-1\end{array}\right)$. The formula of Theorem 2.3 now follows.

3. Congruences for the Rayleigh function. We now show how the formulas of $\$ 2$ can be used in proving congruences for the Rayleigh function.

Let $a / b$ be a rational number, $a$ odd, $b$ even, $b=(2 k+1) 2^{t}$, and let $y=2 n+1+(1-2 n) t-m$, where $m$ is the number of nonzero terms in the base 2 expansion of $2 n$. In [2] the following results were proved.

LEMMA 3.1. If $2 n=2^{e_{1}}+\cdots+2^{e_{m}}, e_{1}>\cdots>e_{m} \geqq 1$, then

$$
2^{v} \sigma_{2 n}(a / b) \equiv 1 \quad(\bmod 2) \text {. }
$$

LEMMA 3.2. If $2 n=2^{e_{1}}, e_{1}>2$, then

$$
2^{y} \sigma_{2 n}(a / b) \equiv 5(2 k+1) / a(\bmod 8) \text {. }
$$

LEMMA 3.3. If $2 n=2^{e_{1}}+\cdots+2^{e_{m}}, e_{i}-e_{i+1}>1$ for $i=1, \cdots$, $m-1$ and $e_{m} \geqq 1$, then

$$
2^{y} \sigma_{2 n}(a / b) \equiv(-1)^{m+1}(2 k+1) / a(\bmod 4) .
$$

We can now prove the following theorems.

THEOREM 3.1. If $2 n=2^{e_{1}}+2^{e_{2}}, e_{1}-e_{2}>2, e_{2}>2$, then

$$
2^{y} \sigma_{2 n}(a / b) \equiv 7(2 k+1) / a(\bmod 8) \text {. }
$$

Proof. From (1.2) and Lemmas 3.1, 3.2 and 3.3, we have 


$$
\begin{aligned}
2^{y} \sigma_{2 n}(a / b) & \equiv[f(2 n, 0)+6 f(2 n, 1)+4(f(2 n, 2)-1)+2](2 k+1) / a \\
& \equiv 7(2 k+1) / a(\bmod 8) .
\end{aligned}
$$

ThEOREM 3.2. If $2 n=2^{e_{1}}+2^{e_{2}}+2^{e_{3}}, e_{i}-e_{i+1}>2$ for $i=1,2$ and $e_{3}>2$, then

$$
2^{y} \sigma_{2 n}(a / b) \equiv 5(2 k+1) / a(\bmod 8) .
$$

Proof. We have

$$
\begin{aligned}
2^{y} \sigma_{2 n}(a / b) & \equiv[3 f(2 n, 0)+2 f(2 n, 1)+4 f(2 n, 2)+4](2 k+1) / a \\
& \equiv 5(2 k+1) / a(\bmod 8) .
\end{aligned}
$$

THEOREM 3.3. If $2 n=2^{e_{1}}+\cdots+2^{e_{m}}, e_{i}-e_{i+1}>2$ for $i=1, \cdots$, $m-1$ and $e_{m}>2$, then

$$
\begin{aligned}
2^{v_{\sigma_{n}}(a / b)} & \equiv 7(2 k+1) / a \quad(\bmod 8) \quad \text { if } m \text { is even, } \\
& \equiv 5(2 k+1) / a \quad(\bmod 8) \quad \text { if } m \text { is odd. }
\end{aligned}
$$

Proof. The proof is by induction on $m$. The theorem is true for $m=1,2,3$. Assume it is true for $1,2,3, \cdots, m-1$. If $m$ is even we have

$2^{y_{\sigma_{2 n}}(a / b)} \equiv[f(2 n, 0)+6 f(2 n, 1)+4 f(2 n, 2)](2 k+1) / a(\bmod 8)$.

We know

$$
\begin{aligned}
& f(2 n, 0)=2^{m-1}-1 \equiv 7 \quad(\bmod 8) \\
& f(2 n, 1)=m 2^{m-2} \equiv 0 \quad(\bmod 4) \\
& f(2 n, 2)=m 2^{m-1}+\left(\begin{array}{l}
m \\
2
\end{array}\right) 2^{m-3} \equiv 0 \quad(\bmod 2)
\end{aligned}
$$

Therefore

$$
2^{y} \sigma_{2 n}(a / b) \equiv 7(2 k+1) / a(\bmod 8) .
$$

If $m$ is odd we have

$$
\begin{aligned}
2^{y_{\sigma_{2 n}}(a / b)} & \equiv[3 f(2 n, 0)+2 f(2 n, 1)+4 f(2 n, 2)](2 k+1) / a \\
& \equiv 5(2 k+1) / a(\bmod 8) .
\end{aligned}
$$

We can use the method of this section to prove congruences (mod 16). The following theorem is first verified for $m=1,2,3,4$ and then proved by induction on $m$.

TheOREM 3.4. If $2 n=2^{e_{1}}+\cdots+2^{e_{m}}, e_{i}-e_{i+1}>3$ for $i=1, \cdots$, $m-1$ and $e_{m}>3$, then 


$$
\begin{array}{rlrlr}
2^{y_{\sigma_{2 n}}(a / b)} \equiv 5((2 k+1) / a)^{3} & (\bmod 16) & \text { if } m \equiv 3 & (\bmod 4), \\
& \equiv 7((2 k+1) / a)^{3} & (\bmod 16) & \text { if } m \equiv 2 & (\bmod 4), \\
& \equiv 13((2 k+1) / a)^{3} & (\bmod 16) & \text { if } m \equiv 1 & (\bmod 4), \\
& \equiv 15((2 k+1) / a)^{3} & (\bmod 16) & \text { if } m \equiv 0 & (\bmod 4) .
\end{array}
$$

4. Relationship between $f(z, s)$ and binomial coefficients. We use the notation of the introduction in this section. Consider the binomial coefficient $\left(\begin{array}{c}2 \\ r\end{array}\right), 0<r \leqq z / 2$. It is well known that the exponent of the highest power of 2 dividing $\left(\begin{array}{l}z \\ r\end{array}\right)$ is $s=v+w-m$. This follows from the fact that if

$$
n=2^{a_{1}}+\cdots+2^{a_{i}}, \quad a_{1}>\cdots>a_{i} \geqq 0,
$$

then the exponent of the highest power of 2 dividing $n$ ! is $n-i$ $[1$, p. 61$]$. Hence, by the definition of $f(z, s)$, we see that $f(z, s)$ is equal to the number of binomial coefficients $\left(\begin{array}{l}z \\ r\end{array}\right), 0<r \leqq z / 2$, divisible by $2^{s}$ but not by $2^{s+1}$. I am grateful to the referee and to L. Carlitz for suggestions concerning this section.

\section{REFERENCES}

1. P. Bachmann, Niedere Zahlentheorie. Erster Teil; Zweiter Teil: Additive Zahlentheorie, reprint, Chelsea, New York, 1968. MR 39 \#25.

2. F. T. Howard, A property of the Rayleigh function, Proc. Amer. Math. Soc. 25 (1970), 719-723.

3. N. Kishore, The Rayleigh function, Proc. Amer. Math. Soc. 14 (1963), 527-533. MR 27 \#1633.

4. - The Rayleigh polynomial, Proc. Amer. Math. Soc. 15 (1964), 911-917. MR $29 \# 6079$.

5. J. Riordan, $A n$ introduction to combinatorial analysis, Wiley, New York; Chapman \& Hall, London, 1958, MR 20 \#3077.

Wake Forest University, Winston-Salem, North Carolina 27109 